\title{
Multiple-Point Mass Flux Measurement System Using Rayleigh Scattering
}

\author{
Amy F. Mielke* \\ NASA Glenn Research Center, Cleveland, Ohio, 44135 \\ Kristie A. Elam ${ }^{\dagger}$ \\ Jacobs Sverdrup, Cleveland, Ohio, 44135 \\ Michelle M. Clem* \\ NASA Glenn Research Center, Cleveland, OH 44135
}

\begin{abstract}
[Abstract] A multiple-point Rayleigh scattering diagnostic is being developed to provide mass flux measurements in gas flows. Spectroscopic Rayleigh scattering is an established flow diagnostic that has the ability to provide simultaneous density, temperature, and velocity measurements. Rayleigh scattered light from a focused 18 Watt continuous-wave laser beam is directly imaged through a solid Fabry-Perot etalon onto a CCD detector which permits spectral analysis of the light. The spatial resolution of the measurements is governed by the locations of interference fringes, which can be changed by altering the etalon characteristics. A prototype system has been used to acquire data in a Mach 0.56 flow to demonstrate feasibility of using this system to provide mass flux measurements. Estimates of measurement uncertainty and recommendations for system improvements are presented.
\end{abstract}

\section{Nomenclature}

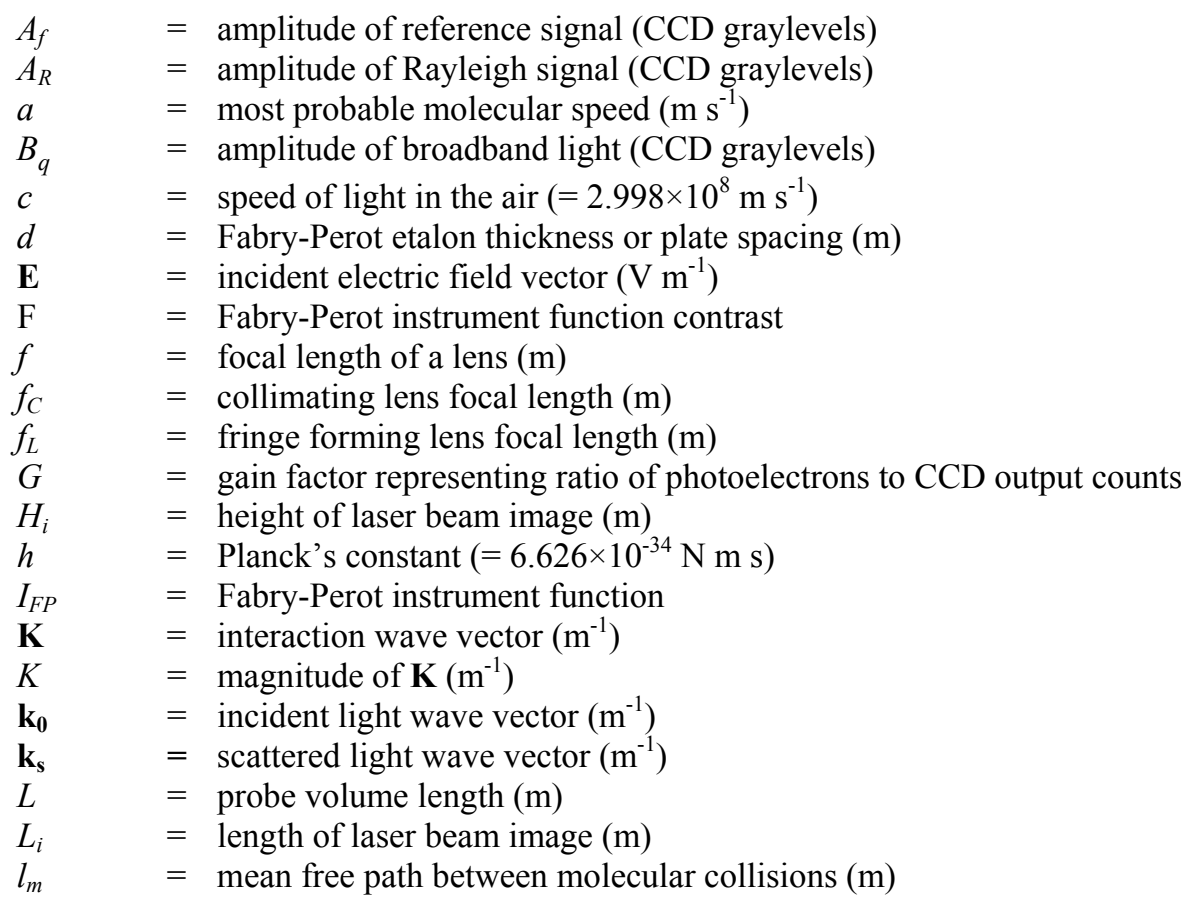

\footnotetext{
${ }^{*}$ Research Engineer, Optical Instrumentation and NDE Branch, 21000 Brookpark Rd/M.S. 77-1, AIAA Member ${ }^{\dagger}$ Optics Technician, 21000 Brookpark Rd/M.S. 77-3

* Research Engineer, Optical Instrumentation and NDE Branch, 21000 Brookpark Rd/M.S. 77-1
} 


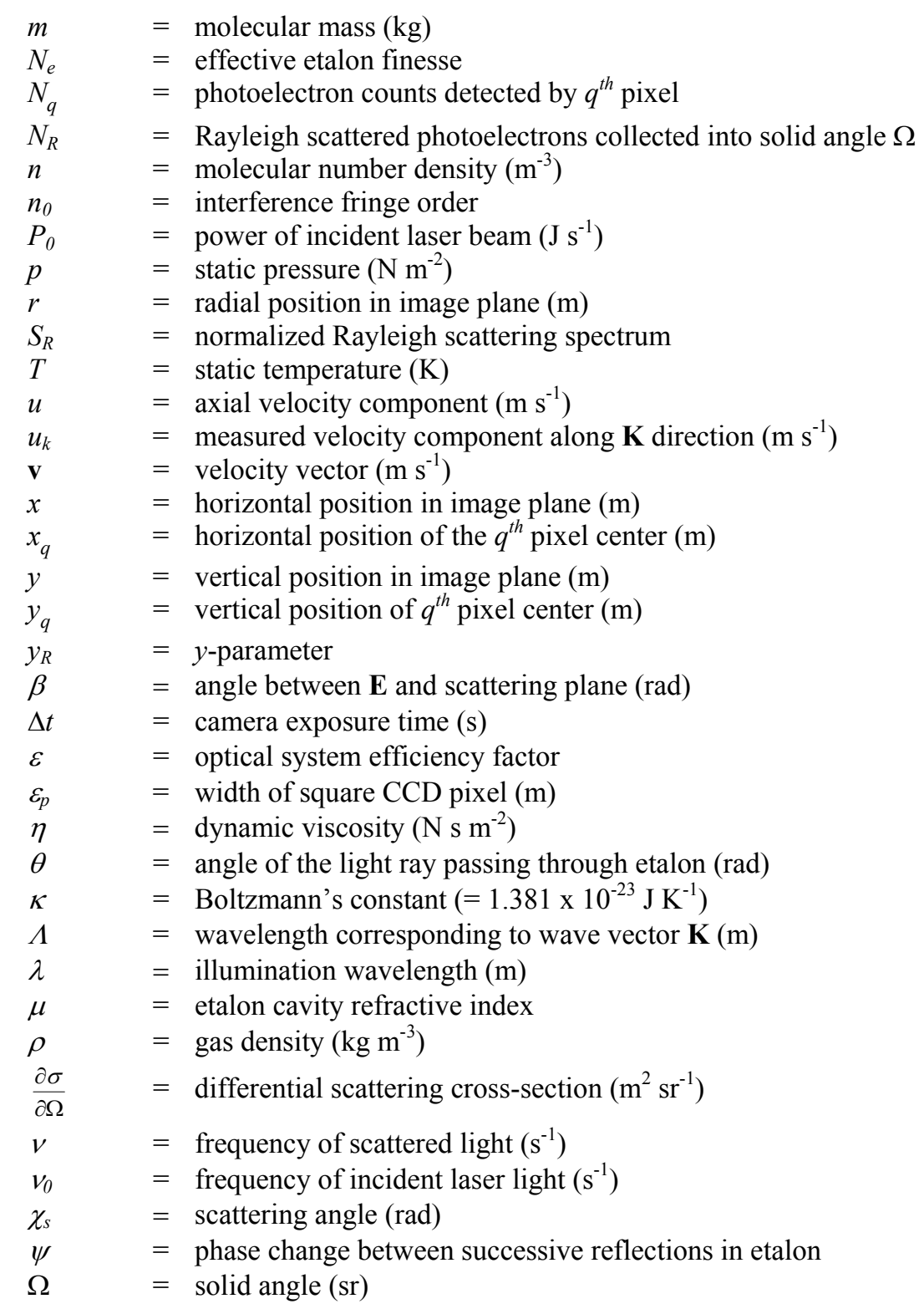

\section{Introduction}

D AYLEIGH scattering has been used in many different ways to provide non-intrusive density, velocity, and/or temperature measurements in various flow environments. Rayleigh scattering is the elastic scattering of light from molecules where the signal strength, Doppler frequency shift, and spectral linewidth of the scattered light provide measurements of density, velocity, and temperature, respectively ${ }^{1}$. Since no energy exchange takes place between the photons and molecules, any laser frequency is applicable, although shorter wavelengths are typically desired since the scattering strength scales by $\lambda^{-4}$. In a non-spectroscopic approach, Rayleigh scattering has been used to measure density and temperature in flames by a direct intensity measurement and applying the ideal gas law; however assumptions must be made about the local gas composition and pressure in this type of work. This nonspectroscopic approach requires that the molecular scattering cross-section not vary greatly in the flow and it is only applicable in clean, particle-free flows. One example of such work is an experiment in which two-point temperature measurements were made at a rate of $10 \mathrm{kHz}$ in a turbulent non-premixed jet flame using a pulsed Nd:YAG laser ${ }^{2}$.

Rayleigh techniques that take advantage of the spectral content of the scattered light to provide flow measurements do so in a variety of ways. Typical Rayleigh linewidths are on the order of $1 \mathrm{GHz}$; hence a very narrow linewidth laser $(\sim 5 \mathrm{MHz})$ and an extremely high resolution filter are required to resolve the spectrum. The most common 
techniques involve either the use of atomic or molecular absorption filters (filtered Rayleigh scattering ${ }^{3,4}$ ) or filters based on interference phenomena, such as the Fabry-Perot (FP) interferometer or etalon (interferometric Rayleigh scattering) to resolve the spectrum of the light. Atomic or molecular absorption filters that have a sharp edge and smooth transition between their minimum and maximum transmission are useful for measuring the frequency shifts and spectral linewidths typically encountered in molecular scattering experiments. Iodine vapor filters are often used because of their strong absorption features in the visible with transitions from strong absorption to full transmission for frequency ranges of $\pm 1 \mathrm{GHz}\left( \pm 0.03 \mathrm{~cm}^{-1}\right)$. Molecular filters are also useful for filtering laser light scattered from surfaces in Rayleigh scattering experiments in wind tunnels or other enclosed experiment chambers 5 .

Interferometric Rayleigh scattering, which is the approach taken in this work, utilizes a Fabry-Perot etalon to resolve the Rayleigh lineshape. A Fabry-Perot etalon consists of two parallel planar reflective plates or surfaces and is typically used in the imaging mode (constant spacing between reflective surfaces) for interferometric Rayleigh scattering ${ }^{6}$. An etalon may be air-spaced or consist of a solid transparent optical material. When light is imaged through the etalon an interference pattern results which is a function of the spectrum of the light convolved with the instrument function of the Fabry-Perot. The Fabry-Perot instrument function is the well-known Airy function. A model function utilizing a Rayleigh spectrum model and the Airy function is fit to the recorded interference pattern to provide flow measurements.

In previous work where time-averaged measurements were acquired using a CCD detector to record the interference pattern $^{7}$ the Rayleigh signal was transmitted to the to the interferometer and detection equipment by an optical fiber. This allows the FP interferometer to be remotely located from the harsh environments commonly encountered in test facilities. This is critical when using an air-spaced etalon that does not have a rigid fixed spacing since vibrations can change the plate spacing and corrupt the spectral measurements. Using an optical fiber to collect the Rayleigh scattered light provides point-wise or spatially-averaged measurements over a finite distance (on the order of 1.0 $\mathrm{mm}$ ) along a focused laser beam. Our research group at NASA Glenn Research Center has developed several fibercoupled point-wise Rayleigh scattering measurement systems that are capable of acquiring data at high sampling rates using high quantum efficiency detectors ${ }^{8-10}$. Although single-point time-resolved measurements are quite useful, there are many situations that would benefit from multiple-point measurements, especially if these can be acquired at high sampling rates. Spatially-resolved measurements have been obtained by imaging scattered light from a laser line or sheet directly through the etalon ${ }^{5,11,12}$.

A spatially-resolved Rayleigh measurement approach is investigated in this paper to provide mass flux measurements along a line. The instrumentation under development is planned for use in the $15 \mathrm{~cm} \mathrm{x} 15 \mathrm{~cm}$ supersonic wind tunnel at NASA Glenn Research Center. In order to provide spatially-resolved measurements using direct-imaging of the laser beam a solid Fabry-Perot etalon is used rather than the adjustable air-spaced FP interferometer used in previous work ${ }^{8-10}$ to avoid degradation of the signal due to vibration of the individual plates in the harsh environment of the facility. For this preliminary work at low data acquisition rates, a low read-noise CCD camera is used to record the image of an 18 Watt $532 \mathrm{~nm}$ laser beam filtered by the FP etalon. The $25 \mathrm{~mm}$ diameter solid fused silica etalon has a $9 \mathrm{GHz}$ free spectral range (FSR) and finesse of about 30 ( $90 \%$ reflectivity coatings). This system will eventually be used to measure flows where shocks are present. The presence of shocks will affect the laser propagation direction, which will change the component of the velocity that is measured and may also move the laser beam out of the depth of focus of the imaging system. The effect of shocks on the measurement system will be analyzed in detail in future work.

\section{Rayleigh Scattering and Fabry-Perot Interferometry}

In molecular Rayleigh scattering an incident electric field interacts with an atom or a molecule inducing a dipole moment that oscillates and radiates at the frequency of the incident field. It is considered an elastic scattering process because the internal energy of the molecule is unchanged and the frequency of the light is changed only by the Doppler effect due to the thermal as well as the bulk motion of the molecules ${ }^{1}$. The frequency spectrum of the scattered light contains information about the gas density, bulk velocity, and temperature. Figure 1 shows a Rayleigh scattering spectrum containing the narrow laser line and a typical Rayleigh spectral peak to illustrate how the flow property measurements are obtained from the spectral information. If the gas composition is fixed, the total intensity of the Rayleigh scattered light is directly proportional to the gas density. The frequency shift between the laser peak 
and the Rayleigh peak is proportional to the bulk flow velocity. The width of the spectrum is related to the gas temperature.

The shape of the spectrum is dependent on gas pressure $p$ and temperature $T$, and the scattering angle $\chi_{\mathrm{s}}^{13}$. A nondimensional parameter $y_{R}$, which represents the ratio of the wavelength of the scattering grating $\left(\Lambda=\frac{\lambda}{2 \sin \frac{\chi_{s}}{2}}\right)$ to the mean free path between molecular collisions $\left(l_{m}=\frac{a \eta}{n_{\kappa T}}\right)$, is used to establish spectral shape regimes:

$$
y_{R}=\frac{\Lambda}{2 \pi l_{m}}=\frac{p}{\eta K a}
$$

where

$$
a=\sqrt{\frac{2 \kappa T}{m}}
$$

The measured velocity component, $u_{k}$, is in the same direction as the interaction wave vector $\mathbf{K}$, which is the bisector of the incident and scattered light wave vectors, $\mathbf{k}_{\mathbf{0}}$ and $\mathbf{k}_{\mathbf{s}}$, respectively (Fig. 2). The interaction wave vector and its magnitude $K$ are given by:

$$
\begin{gathered}
\mathbf{K}=\mathbf{k}_{\mathbf{s}}-\mathbf{k}_{\mathbf{0}} \\
K=|\mathbf{K}|=\frac{4 \pi}{\lambda}\left[\sin \frac{\chi_{s}}{2}\right]
\end{gathered}
$$

The geometry of the optical arrangement in an experiment is designed such that the desired component of the velocity vector $\mathbf{v}$ is measured:

$$
u_{k}=\frac{\mathbf{K} \cdot \mathbf{v}}{K}
$$

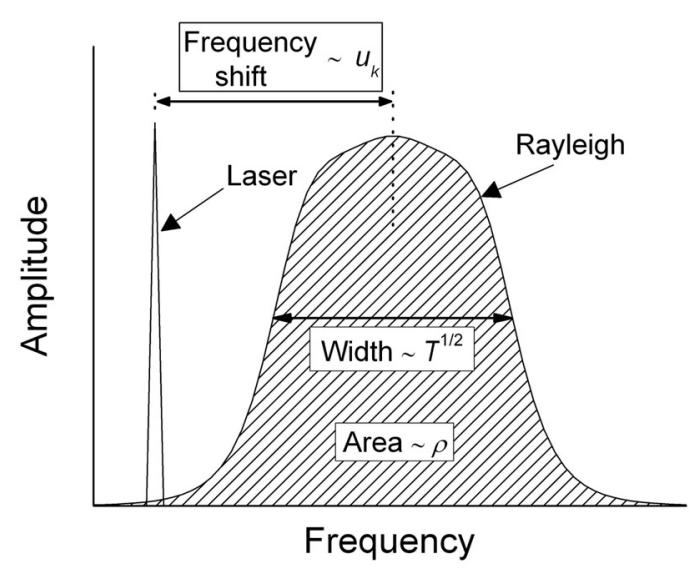

Fig. 1 Rayleigh scattering spectrum.

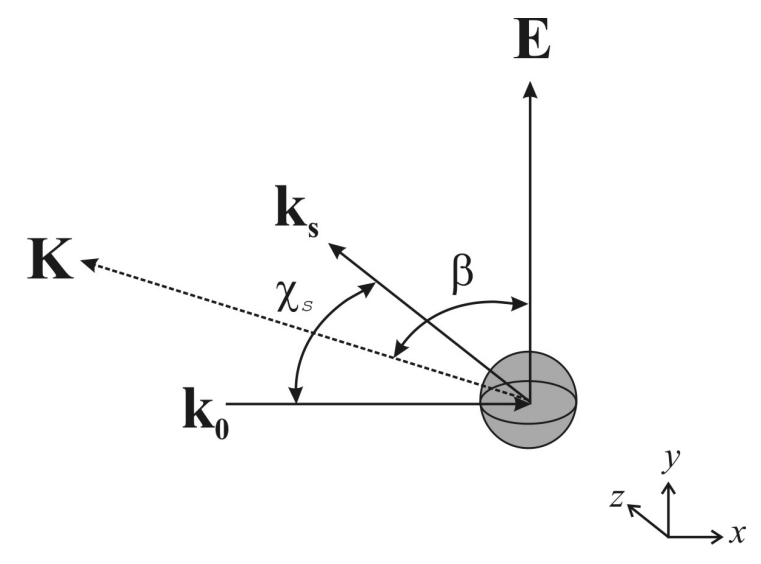

Fig. 2 Light scattering from a moving particle. 
The spectrum of the Rayleigh scattered light is analyzed using a planar solid Fabry-Perot (FP) etalon (Fig. 3) ${ }^{6}$. A solid FP etalon consists of an optical material with parallel planar surfaces having a reflective coating. Maximum transmission occurs when the optical path-length difference $(O P D)$ between each transmitted beam is equal to an integer multiple of the wavelength, or

$$
O P D=2 \mu d \cos \theta=m_{i} \lambda
$$

where $m_{i}$ are integer values representing the order of interference. Maximum reflectivity or minimum transmission occurs when the $O P D$ is equal to half of an odd multiple of the wavelength. By varying either the etalon thickness $(d)$, the index of refraction $(\mu)$ of the optical medium in the cavity between the reflective surfaces, or the angle of the light ray passing through the FP etalon $(\theta)$ transmission of different frequencies will occur and recreate the Rayleigh spectrum. When $\theta$ is varied by imaging points off-axis the solid FP etalon is said to be operated in the static imaging mode. A lens at the etalon output images the light source at the image plane where a detector is located, as shown in Fig. 3. If the incoming light has a very narrow linewidth the imaged intensity pattern is essentially a delta function convolved with the Fabry-Perot instrument function, which is defined as:

$$
I_{F P}=\frac{1}{1+F \sin ^{2} \frac{\psi}{2}}
$$

where $F$ is the contrast:

$$
F=\frac{1}{\sin ^{2} \frac{\pi}{2 N_{e}}}
$$

$N_{e}$ is the effective etalon finesse and $\psi$ is the phase change of the light between successive reflections

$$
\psi=\frac{4 \pi \mu d}{\lambda} \cos \theta
$$

Figure 4 shows a typical fringe pattern when a planar single-frequency light source is imaged through the FabryPerot etalon. For spectrally broadened light, such as Rayleigh scattered light, the fringes broaden accordingly; the linewidth of the fringes provides a measure of temperature. The frequency shift of the light, which is associated with the bulk flow velocity, is determined by a spatial shift in the fringe positions in the image. In this work, the light source is molecular scattered light from a focused laser beam, which provides a linear interference pattern, such as that shown in Fig. 5. Flow measurements are obtained only where a fringe is located, which determines the spatial resolution of the measurements in the flow field. The locations of the fringes can be adjusted by changing the etalon thickness $d$ and/or the medium refractive index $\mu$. For a standard solid etalon, the thickness and refractive index can be adjusted by changing the etalon temperature. A method of controlling the fringe locations will be developed in future work.

Rayleigh scattered light is collected from a finite length $L$ of the laser beam and imaged at the CCD detector. The expected number of Rayleigh photoelectron counts incident on the detector plane without the FP etalon in the optical path can be expressed as:

$$
\left\langle N_{R}\right\rangle=\int_{\Delta \Omega} \frac{\varepsilon P_{0} n L \lambda \Delta t \frac{\partial \sigma}{\partial \Omega} \sin ^{2} \beta}{h c} \partial \Omega
$$

where $\varepsilon$ is the overall system efficiency including detector quantum efficiency and other losses. With the interferometer in the optical path, the amount of energy, in terms of CCD gray level counts, collected on the $q^{\text {th }}$ pixel 
of the detector centered at position $\left(x_{q}, y_{q}\right)$ including light scattered from gas molecules (Rayleigh scattering), stray scattered light at the laser frequency, and broadband background light is expressed as:

$$
\left\langle N_{q}\right\rangle=\frac{A_{R}}{\varepsilon_{p}^{2}} \int_{y_{q}-\frac{\varepsilon_{p}}{2}}^{y_{q}+\frac{\varepsilon_{p}}{2}} \int_{x_{q}-\frac{\varepsilon_{p}}{2}}^{x_{q}+\frac{\varepsilon_{p}}{2}} \int_{-\infty}^{+\infty} S_{R}\left(v, u_{k}\right) I_{F P}(v, x, y) d v d x d y+\frac{A_{f}}{\varepsilon_{p}^{2}} \int_{y_{q}-\frac{\varepsilon_{p}}{2}}^{y_{q}+\frac{\varepsilon_{p}}{2}} \int_{x_{q}-\frac{\varepsilon_{p}}{2}}^{x_{q}+\frac{\varepsilon_{p}}{2}} I_{F P}\left(v_{0}, x, y\right) d x d y+B_{q}
$$

where the amplitude of the Rayleigh scattered light is defined as:

$$
A_{R}=\left(\left\langle N_{R}\right\rangle \varepsilon_{p}^{2}\right) /\left(G L_{i} H_{i}\right)
$$

The second and third terms in Eq. (11) are associated with scattered light at the laser frequency and background light, respectively. The Rayleigh spectrum $S_{R}$ is evaluated using the TENTI S6 kinetic theory model ${ }^{13,14}$. The imaged fringe patterns are analyzed by maximum likelihood estimation (MLE) analysis using the model function described in Eq. (11) to extract the density and velocity information at multiple spatial points in the flow field simultaneously.

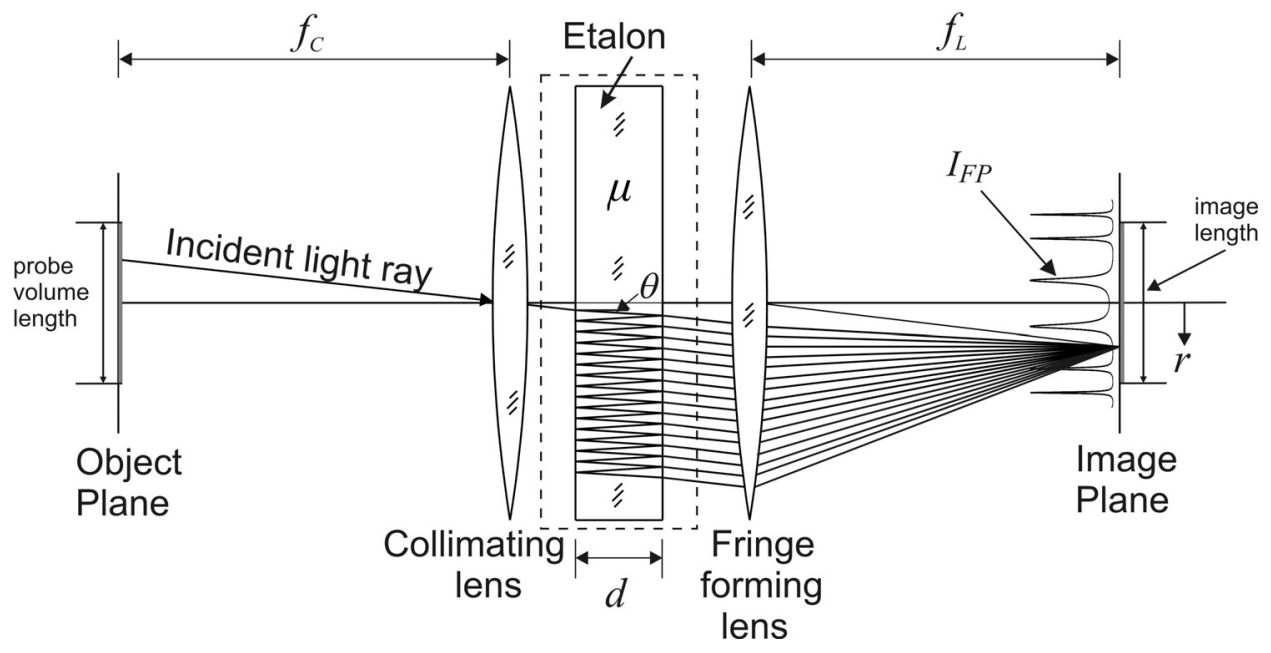

Fig. 3 Basic optical arrangement for spectrally-analyzing a uniform light source using a solid Fabry-Perot etalon.

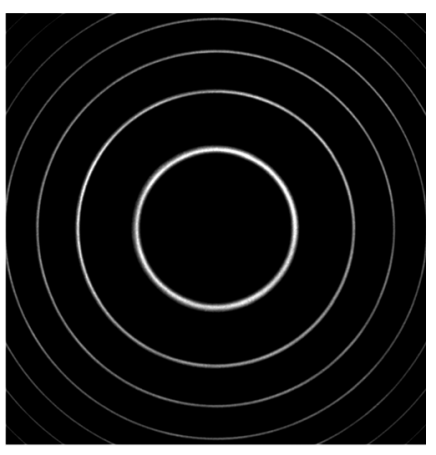

Fig. 4 Concentric ring interference pattern from a planar singlefrequency light source imaged through a Fabry-Perot etalon.

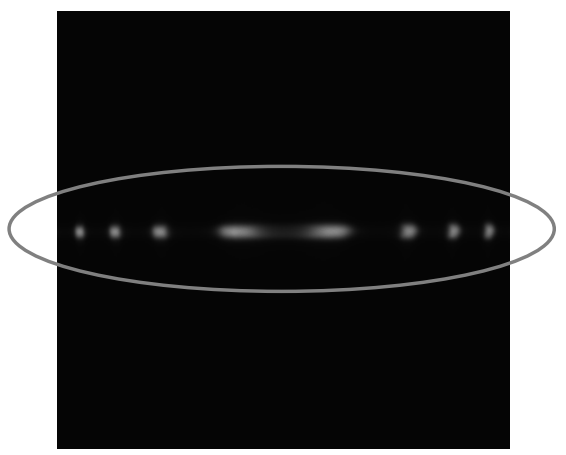

Fig. 5 Rayleigh light scattering from a focused single-frequency laser beam imaged through a Fabry-Perot etalon. 


\section{Experiment}

\section{A. Rayleigh scattering mass flux measurement system}

Figure 6 shows a diagram of the prototype mass flux measurement system arranged around a free jet equipped with a 10-mm diameter convergent nozzle. Since the measurement technique relies on having particle-free gas flow, a series of sub-micron filters were placed in line with the air plumbing to remove dust, oil and water from the air supply prior to entering the nozzle flow system. A Coherent Verdi $18 \mathrm{~W}$ continuous-wave 532-nm wavelength $\mathrm{Nd}$ :Vanadate laser with a $2.25-\mathrm{mm}$ diameter output beam and $5 \mathrm{MHz}$ linewidth provided the incident light for the system. The vertically-polarized laser beam was focused with a $250-\mathrm{mm}$ focal length lens (L1) to a $75-\mu \mathrm{m} 1 / \mathrm{e}^{2}$ diameter at the probe volume. The beam was oriented at a $45^{\circ}$ angle to the primary flow direction and light was collected at a $90^{\circ}$ scattering angle. The incident and scattering wave vectors were arranged such that the axial component of the jet velocity $u$ was measured, as indicated by the red $\mathbf{K}$ vector in Fig. 6 . A 4.3 -mm length of the laser beam was imaged through the solid FP etalon onto the detector of a Princeton Instruments VersArray backilluminated, scientific-grade CCD camera. A controlled amount of stray light scattered from surfaces was also imaged through the etalon to provide reference laser light in the acquired images. The presence of both incident laser light and Rayleigh scattered light in the image greatly reduced velocity measurement error due to assumptions about the laser frequency since this particular laser has short-term frequency drift of $\pm 10 \mathrm{MHz}$ that could potentially lead to $\pm 4 \mathrm{~m} / \mathrm{s}$ velocity bias error ${ }^{15}$. Two $f / 4200-\mathrm{mm}$ focal length lenses (L2 \& L3) provided 1:1 imaging of the laser beam at image plane 1 and a 100-mm focal length lens (L4) collimated the light collected from this intermediate laser beam image through the etalon. The $25-\mathrm{mm}$ diameter etalon was made of fused silica with a refractive index of approximately 1.46 . The etalon had $90 \%$ reflectivity coatings providing a reflective finesse of 30 . The etalon was approximately $11.7-\mathrm{mm}$ thick giving a FSR of $8.8 \mathrm{GHz}$. A Nikon $/ 4300-\mathrm{mm}$ focal length lens focused the interference fringes at the detector of the CCD camera (image plane 2) providing a magnification factor of 2.85 . The $512 \times 512$ imaging array had a $100 \%$ fill factor, $24 \times 24-\mu$ m pixels, 16 -bit dynamic range, high quantum efficiency, thermoelectric cooling and low-noise electronics. Images of the Rayleigh and reference laser light were acquired with 1 second exposure time to ensure that ample signal was obtained in this initial feasibility study; however, shorter exposure times are desired to provide instantaneous snapshots of the flow properties. The images contained 6 unequally-spaced concentric fringes across the $4.3-\mathrm{mm}$ length of the laser beam, which provided 12 measurement points with an average spacing of $0.28-\mathrm{mm}$. The imaged region of the flow was not sufficient to sample the entire jet width in one shot; therefore, the jet was mounted on a single-axis translation stage so that the probe volume could be positioned at various radial locations in the jet plume. The probe volume was positioned axially at three jet diameters from the nozzle exit and was scanned at 1-mm increments across the flow to provide full radial profiles of the jet plume.

A sample image for a flow velocity of approximately $190 \mathrm{~m} / \mathrm{s}$ is shown in Fig. 7. The full circular fringes are associated with reference laser light while the Rayleigh signal is only present along the image of the laser beam, which runs horizontally through the center of the fringe pattern. Vertical and horizontal line profiles are plotted illustrating the reference signal and the combined Rayleigh and reference signals, respectively. The amplitude of the reference signal varies across the image since no effort was made to provide a uniform source of reference light. The amplitude of the reference light varies between $16 \%$ and $27 \%$ of the total combined signal. MLE analysis of the interference pattern provides density and velocity measurements at each location where a fringe is present. The fringes are not equally spaced, but the measurements in a single image have an average spatial resolution of 0.28$\mathrm{mm}$, as set by the fringe spacing. 


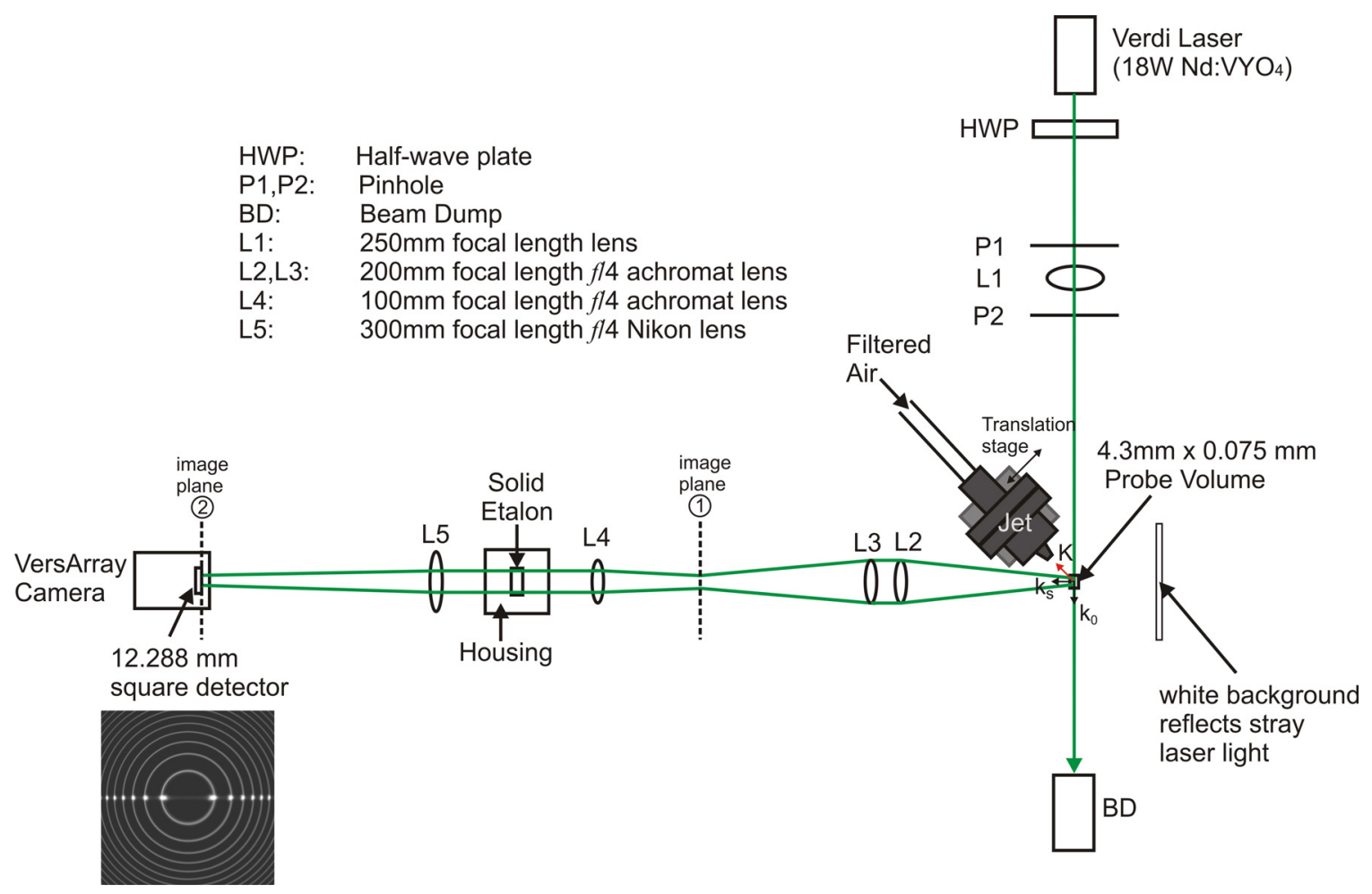

Fig. 6 Schematic of Rayleigh mass flux measurement system arranged to measure the axial velocity component of a free jet flow. The jet is mounted on a 1-axis translation stage to move the location of the probe volume in the flow.

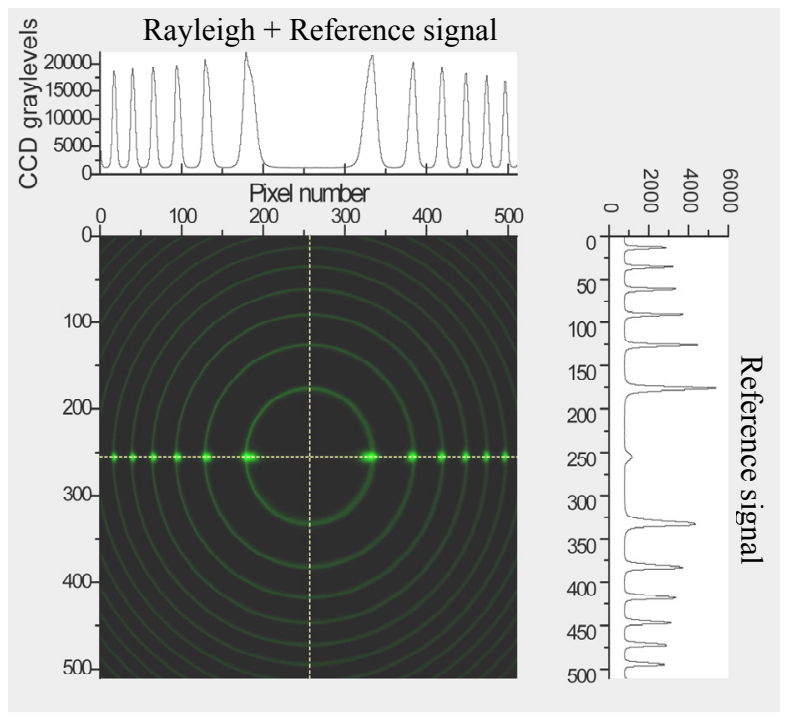

Fig. 7 Typical Fabry-Perot interference fringe image acquired in this experiment. The probe volume was located in the jet core where the velocity was approximately $190 \mathrm{~m} / \mathrm{s}$. The vertical and horizontal line profiles illustrate the reference laser signal and the combined Rayleigh and reference signals, respectively. 


\section{B. Data analysis method}

The reference laser fringes were analyzed by MLE to obtain more accurate etalon finesse and thickness values since the manufacturer quoted a range of values for these parameters. One other system parameter that required calibration was the optical system efficiency $\varepsilon$. To estimate $\varepsilon$, several images were acquired in the core of the flow where the static and stagnation pressure and stagnation temperature measurements could be used in the isentropic flow relations to estimate the flow properties. The efficiency factor was estimated from MLE by holding the jet flow parameters $(\rho, T, u)$ fixed at the isentropic values. After calibration, MLE analysis using the model function given in Eq. (11) was used to provide estimates of $\rho$ and $u$ from the Rayleigh signal, and $A_{f}$ and the reference fringe radius from the reference signal that was superimposed on the Rayleigh fringe data. Only two adjacent lines of pixels were used in the analysis rather than all pixels spanning the height of the laser beam image because the intensity was not uniform across the entire height and would require additional calibration of the intensity profile of the imaged beam. Therefore, the height of each measurement volume was approximately $0.017-\mathrm{mm}$. The length of each measurement volume varied from $0.05-\mathrm{mm}$ to $0.16-\mathrm{mm}$ depending on fringe width. The simultaneous density and velocity measurements were used to calculate mass flux at the measurement locations. Although temperature estimates were not of interest in this work, the gas temperature affects the width and shape of the spectrum so it was necessary to provide a reasonable estimate of the temperature in the model function. Therefore, the temperature was estimated from the ambient pressure and the gas density estimated from the fringe data using the Ideal Gas Law.

Figure 8 shows a typical profile of interference fringes from this experiment where fringe 1 is the innermost fringe of the circular pattern and fringe 6 is the outermost fringe (only the left half of the fringe pattern is shown). The data is represented by the solid black line and the circular symbols represent the values of the fit to the data using MLE. Figure 9 shows the individual fringes plotted on different scales to better illustrate the goodness of the fits. The reference light is a sharp peak on top of the broader Rayleigh peak, which is denoted by a dashed line in the plots of Fig. 9. The amplitude of the reference light varies from about $6000 \mathrm{CCD}$ counts at fringe 1 to $3000 \mathrm{CCD}$ counts at fringe 6 . Because the $\mathbf{K}$ vector is oriented in the negative axial direction the Rayleigh fringe radius shifts more toward the center of the fringe pattern as velocity increases; therefore the peaks of the Rayleigh fringe are slightly further to the right than the reference fringe peaks in Fig. 9. The model function does not seem to fit very well in the transition region between the Rayleigh and reference peaks. Effects such as flow turbulence and aperture broadening of the spectrum ${ }^{7}$ have not been taken into account in this analysis. Further work will be done to examine the reason for discrepancies between the data and fit values. Also note that each peak has the same spectral width (Full-WidthHalf-Maximum (FWHM) $\sim 1.77 \mathrm{GHz}$ ); however the number of pixels spanned by each fringe reduces as fringe order increases. This leads to coarser frequency resolution at higher fringe orders as shown by the fact that the FWHM of fringe 1 spans 19 pixels ( $\sim 93 \mathrm{MHz} /$ pixel) while the FWHM of fringe 6 only spans 6 pixels ( $\sim 295 \mathrm{MHz} /$ pixel). As a result, the reference peak is not adequately resolved at fringes 5 and 6 where the narrow reference peak only spans approximately 1-2 pixels. A similar problem was encountered in the work of Ref. 12. It appears that the reference and Rayleigh peak locations and the shape of the Rayleigh peak are still recovered well enough in fringes 1-4 to provide reasonable velocity and density estimates for this initial feasibility study; however the accuracy of the velocities from fringes 5 and 6 are greatly reduced since the reference peak location have increased uncertainty.

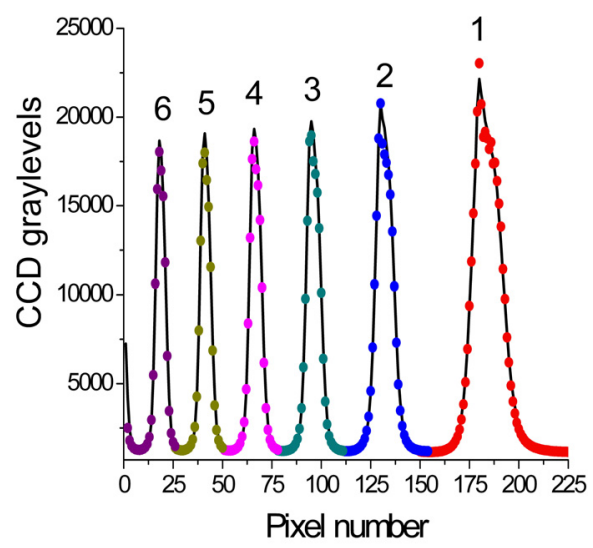

Fig. 8 Data and fit values for an image located in the jet core where the velocity is approximately $190 \mathrm{~m} / \mathrm{s}$. The data is represented by the solid black line. The fit values are shown as various colored round symbols. The different colored symbols are used to differentiate between fit values for each separate fringe. 

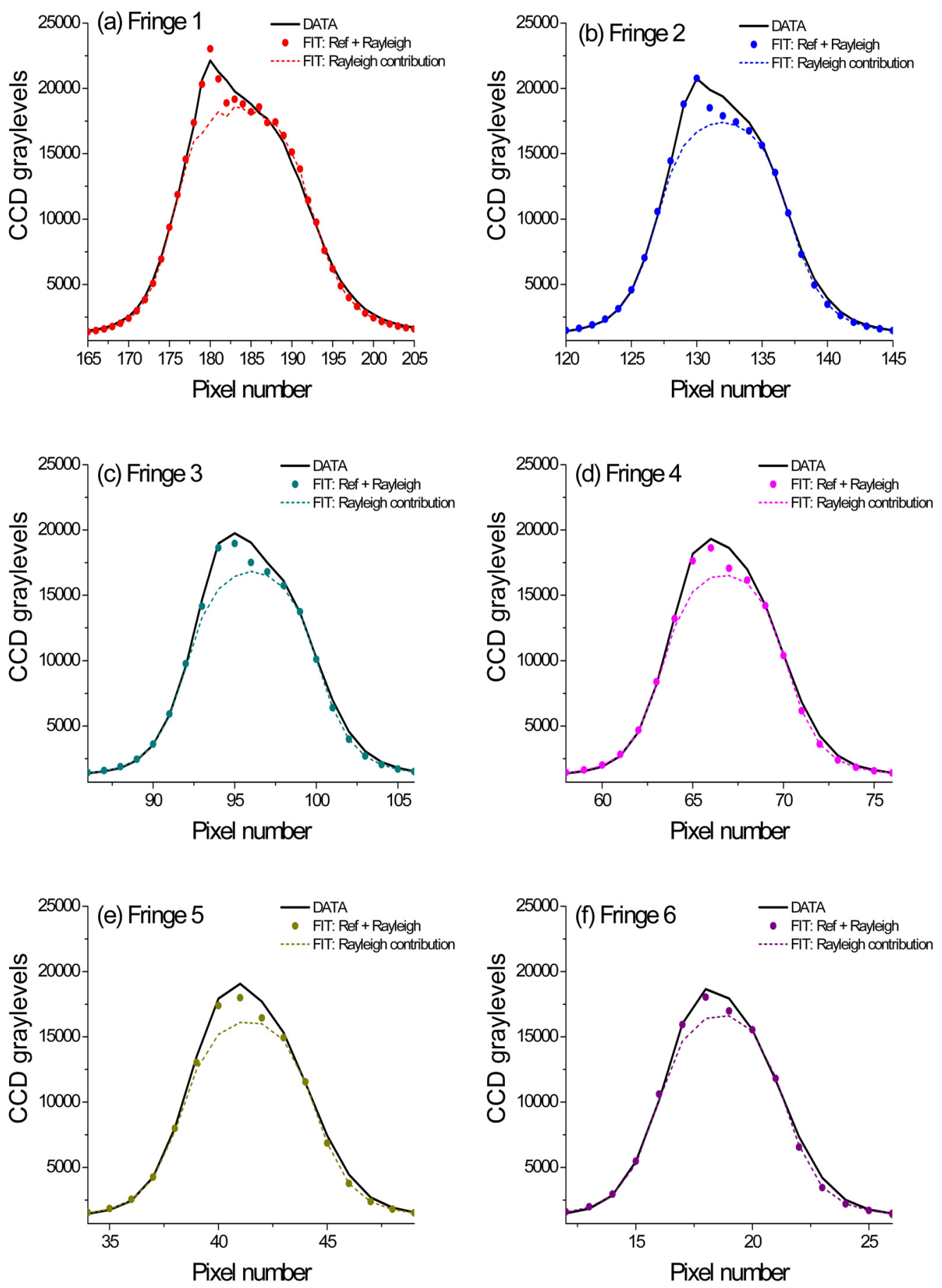

Fig. 9 (a-f) Individual fringe profiles showing the data (solid line) and fit values (solid circles) for each fringe from innermost (a) to outermost (f) fringes. The Rayleigh contribution, as determined by MLE, is shown as a dashed line in each plot. Note that although each peak has the same spectral linewidth $(\sim 1.77$ GHz), the number of pixels spanned decreases as the fringe order increases. The narrow reference peak only spans approximately 1-2 pixels in fringes 5 and 6 , which is not sufficient to accurately detect its location. 


\section{Results and Recommendations}

\section{A. Flow mapping and image quality issues}

A Mach 0.56 air flow issuing from a convergent nozzle flow system was used as an initial validation experiment to demonstrate feasibility of a direct imaging Rayleigh system for mass flux measurements. The probe volume was scanned radially $\pm 10 \mathrm{~mm}$ on either side of the jet axis at $1-\mathrm{mm}$ increments. The acquired images contained six circular fringes which provided twelve measurement locations across the 4.3- $\mathrm{mm}$ imaged length of the laser beam. Unfortunately, a significant portion of the images appeared to be "blurred" as observed in a typical image shown in Fig. 10. The figure shows the centermost fringe where the blurring is most noticeable. The right and bottom regions of the image are not as sharp as the top and left regions of the image. Upon further investigation the source of the problem was determined to be an uneven reflectivity coating on the etalon rather than an out-of-focus issue. The regions of the etalon that produce wider fringes have a lower reflectivity, and hence a lower finesse value, than the regions producing sharp narrow fringes. The wider fringes bring about increased uncertainty in the velocity measurements since it is more difficult to accurately determine the locations of the reference and Rayleigh peaks. Therefore results are only presented for the six fringes on the left side of the images where sharp narrow fringes were recorded. Fig. 11 shows a map of the measurement locations showing the six fringe locations at each station in the radial scan. The laser beam propagation is shown for the first probe location in the radial scan. The nozzle location relative to the probe volume locations is shown for reference.

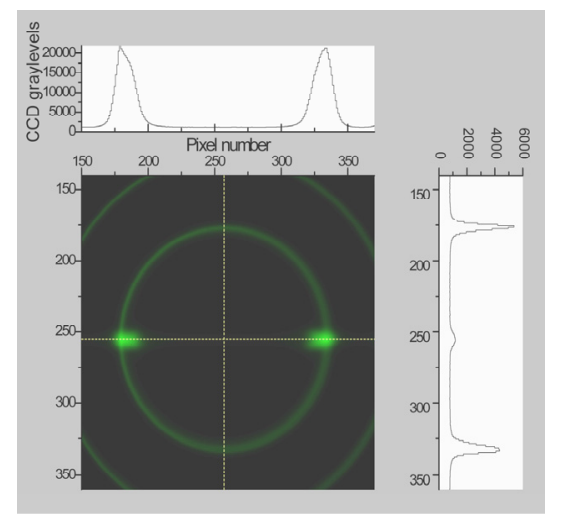

Fig. 10 Sample interference fringe image demonstrating wider fringes in the right and lower regions of the image due to finesse variations across the etalon.

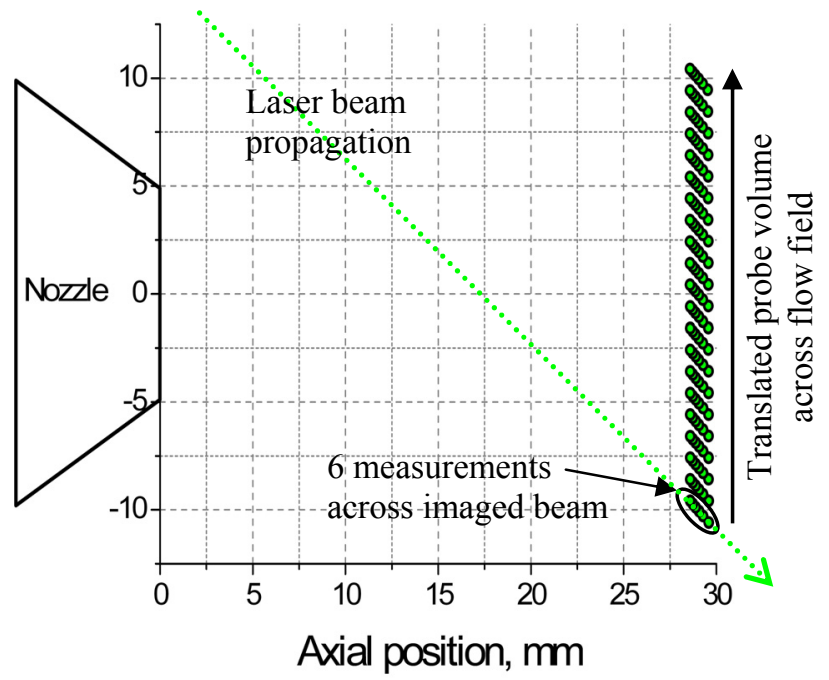

Fig. 11 Map of the measurement locations in the radial scan of the flow field.

\section{B. Measurements in a Mach 0.56 jet plume}

Velocity profiles at the axial locations noted for the six fringes analyzed in this experiment are shown in Fig. 12 along with a dash-dot line indicating the jet exit velocity calculated using isentropic relations. Since the probe volume is located close to the end of the potential jet core the flow exhibits a more parabolic-shaped profile rather than a top-hat profile. The two innermost fringes which have the most number of pixels spanning the width of the fringe provide the most accurate results. The subsequent fringes get narrower as fringe order increases resulting in reduced pixel resolution across the fringe width and less accurate velocity estimates. The accuracy of the velocity estimates from the higher order fringes could be improved by using a CCD detector that could provide higher spatial resolution. Ideally the FWHM of the instrument function should span 3 or more pixels to provide sufficient resolution for identifying the reference peak location. Figure 13 shows the density profiles from this experiment 
along with the corresponding jet exit density and the ambient density, indicated by the black and blue dash-dot lines, respectively. The accuracy of the density estimates from the outermost fringe (fringe 6) is very poor at a few points in the profile. This could be due to a lack of pixel resolution across the fringe or to very fine particles present at those specific measurement locations. When a particle enters the probe volume it only affects the fringe that images the location in the flow where the particle is present. Therefore it is possible that only a single fringe in an image was affected by a passing particle. The particle would increase the signal strength and make it appear that the gas density is much higher than it actually is. The density profiles in Fig. 13 are asymmetric about the jet axis. The position of the jet hardware was translated radially in order to acquire these profiles. As the jet hardware approached the collection optics the hardware intersected the collection cone, blocking some of the scattered light. This produces artificially low density estimates on the right side of the flow profile. If this occurrence cannot be avoided then a varying efficiency factor must be evaluated to account for the reduced light collection. Figure 14 shows the mass flux profiles that were evaluated by taking the product of the Rayleigh velocity and density measurements. The jet exit mass flux is shown by the dash-dot line for reference. The accuracy of the mass flux measurements is limited by the accuracy with which the velocity and density measurements can be obtained.

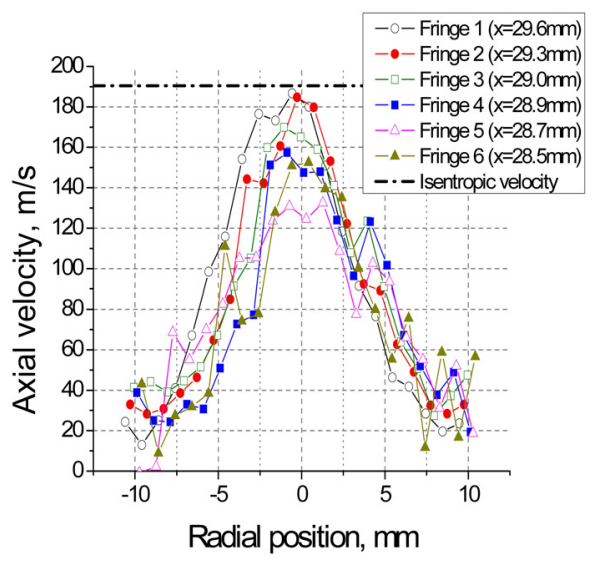

Fig. 12 Radial profiles of the axial velocity component in a Mach 0.56 flow. Each profile is associated with a specific fringe in the interference pattern. The profiles are located between 28.5 and $29.6 \mathrm{~mm}$ downstream of the nozzle exit. The jet exit velocity is indicated by the dash-dot line.

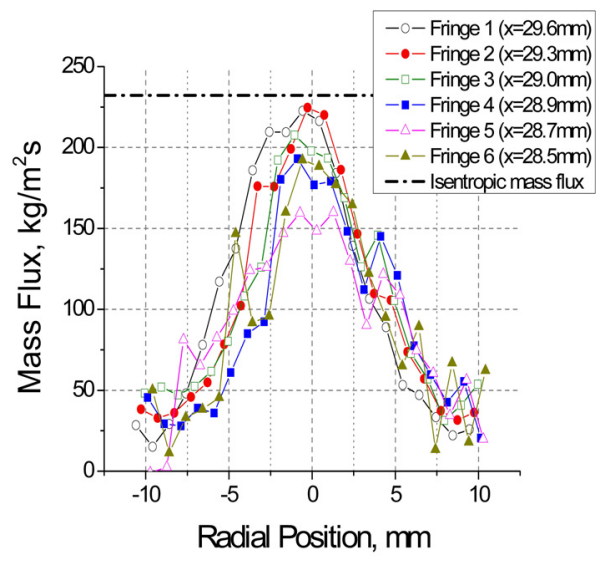

Fig. 14 Radial profile of mass flux calculated from the velocity and density measurements presented in Figs. 12 and 13. The jet exit mass flux is indicated by the dash-dot line.

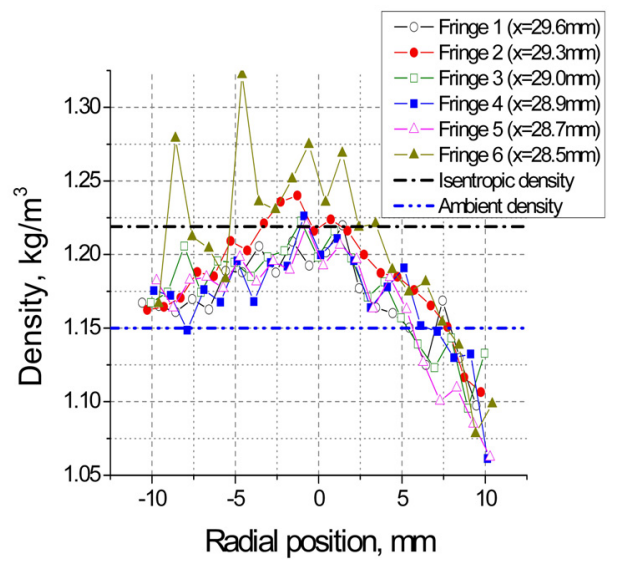

Fig. 13 Radial profiles of gas density in a Mach 0.56 flow for axial locations of 28.5 to 29.6 $\mathrm{mm}$ downstream of the nozzle exit. The jet exit and ambient density values are indicated by the black and blue dash-dot lines, respectively. 


\section{Uncertainty analysis}

A Cramer-Rao lower bound (CRLB) analysis ${ }^{15,16}$ was used to determine the uncertainty in the density and velocity estimates by means of a sensitivity analysis of the model function to small changes in these flow parameters. The CRLB gives the absolute lowest possible variance in the measurements assuming that the only noise is the electronic shot noise in the detection system. The density uncertainty is mostly affected by the Rayleigh signal strength. Since the Rayleigh signal strength does not vary greatly across the flow the uncertainty does not change very much. The density uncertainty was calculated to be on the order of $0.35 \%$ except when the signal was attenuated by the nozzle hardware. The CRLB estimates were used to put error bars on the velocity and mass flux measurements in Figs. 15 and 16, respectively, to show the variation in uncertainty between the innermost and outermost fringes. The uncertainty in the velocity estimates, and hence also the mass flux estimates is much greater for the narrower higher order fringe (fringe 6) due to reduced frequency-per-pixel resolution. Figure 17 shows the velocity uncertainty for Mach 0.56 flow conditions as a function of frequency-per-pixel resolution, where the resolution becomes coarser with increasing fringe order. This figure clearly illustrates an increase in measurement uncertainty as frequency resolution gets coarser since the reference peak cannot be detected as accurately. It is not clear why there is a slight decrease in measurement uncertainty from $250 \mathrm{MHz} /$ pixel to $300 \mathrm{MHz} /$ pixel; however this is consistent with the velocity results shown in Fig. 12 where the $5^{\text {th }}$ fringe, which has $250 \mathrm{MHz} /$ pixel resolution, exhibits worse measurement accuracy than the $6^{\text {th }}$ fringe, which has $300 \mathrm{MHz} /$ pixel resolution.

CRLB analysis was also used to evaluate the velocity measurement uncertainty as a function of total signal level (Fig. 18), reference signal amplitude (Fig. 19) and velocity magnitude (Fig. 20). Figure 18 shows that uncertainty decreases as the total signal strength increases since the signal-to-noise ratio increases. As the signal strength approaches the noise level ( $\sim 700 \mathrm{CCD}$ graylevels in this case) the uncertainty increases significantly. Figure 19 shows that increasing the reference signal amplitude while holding the Rayleigh signal amplitude constant and assuming that the reference fringe radius is known causes the measurement uncertainty to increase. The large jump in uncertainty between having no reference light and a very small amount of reference light (50 CCD graylevels) indicates that the presence of stray laser light degrades the quality of the Rayleigh signal; however without the reference signal the reference fringe radius would not be known to high accuracy, which would increase the uncertainty of the velocity measurements perhaps more than the increase due to added noise from the stray laser light. Figure 20 demonstrates that as velocity magnitude increases the measurement uncertainty decreases. This is expected since the reference and Rayleigh peak become separated by a greater amount making the individual peaks easier to discern.

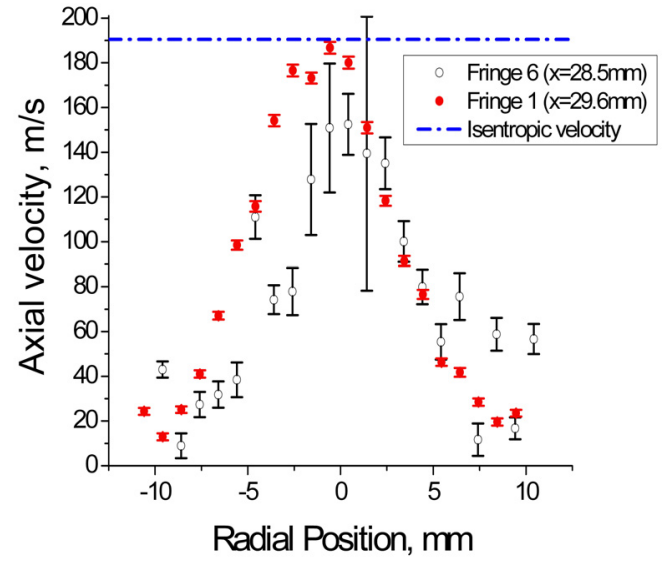

Fig. 15 Axial velocity profiles based on measurements from the innermost (fringe 1) and outermost (fringe 6) fringes in the interference pattern demonstrating the much greater measurement uncertainty (error bars) in the data from fringe 6.

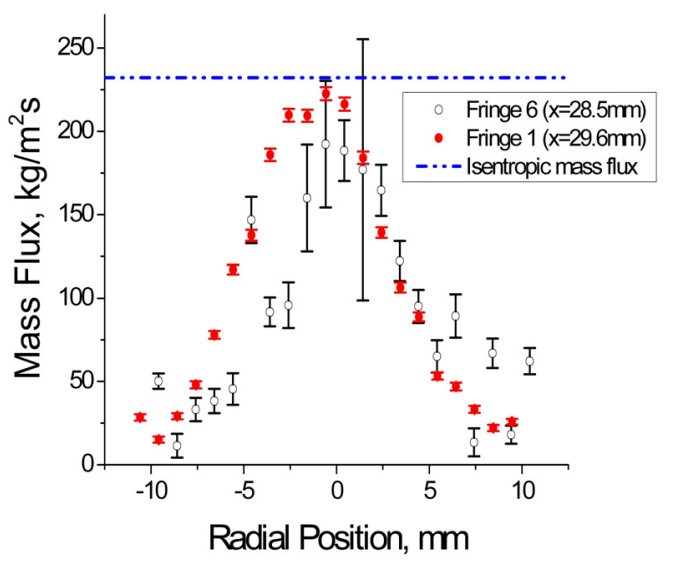

Fig. 16 Mass flux profiles based on measurements from the innermost (fringe 1) and outermost (fringe 6) fringes in the interference pattern demonstrating the measurement uncertainty (error bars) based on the combined uncertainty in both density and velocity measurements used to calculate mass flux. 


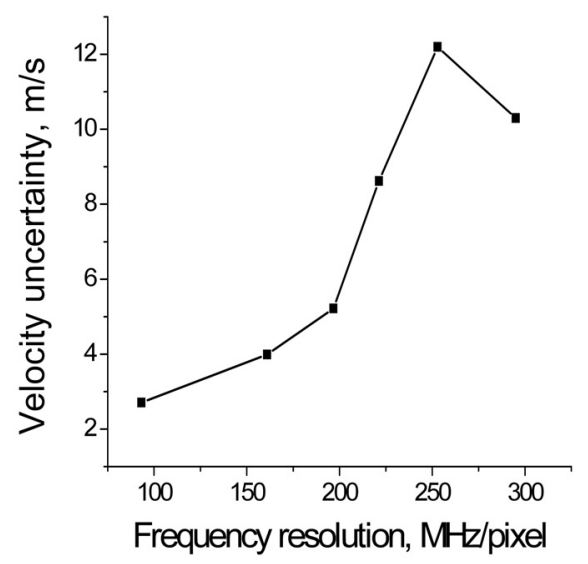

Fig. 17 Velocity uncertainty as a function of frequency resolution in terms of $\mathrm{MHz} /$ pixel where the innermost fringe has the finest resolution and the outermost fringe as the coarsest resolution.

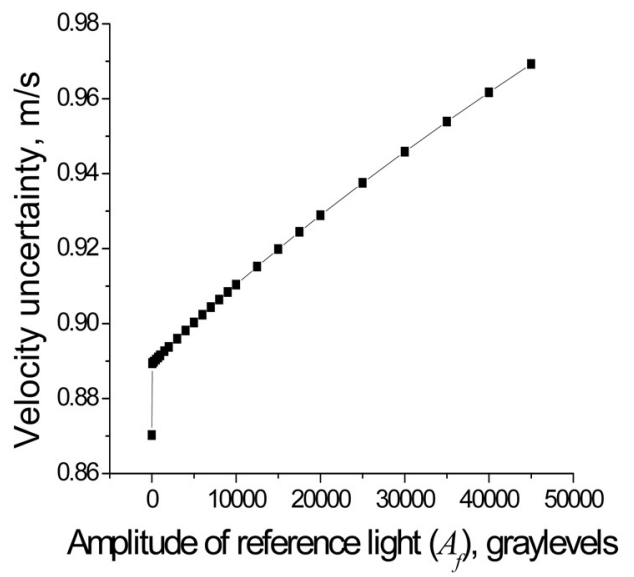

Fig. 19 Velocity uncertainty as a function of reference signal amplitude based on measurements from the innermost fringe. The Rayleigh signal amplitude was held fixed at $A_{R}=16000$, which was the approximate value in the experiments, and the reference fringe radius was held fixed at $1.86-\mathrm{mm}$.

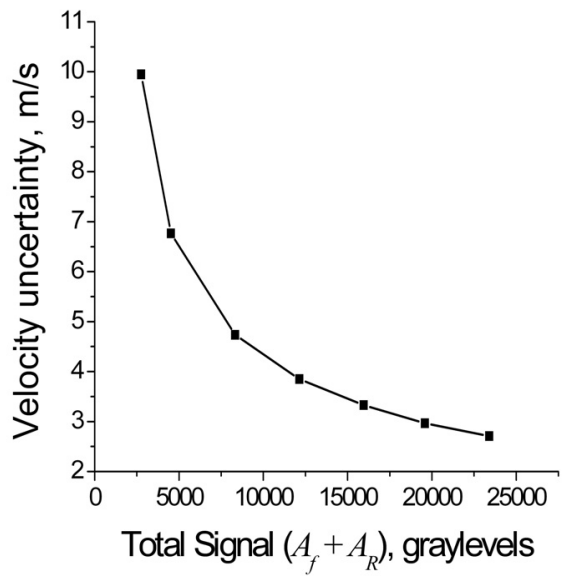

Fig. 18 Velocity uncertainty as a function of total signal amplitude $\left(A_{f}+A_{R}\right)$ based on measurements from the innermost fringe. The ratio of reference signal to Rayleigh signal $\left(A_{f} / A_{R}\right)$ was maintained at 0.375 .

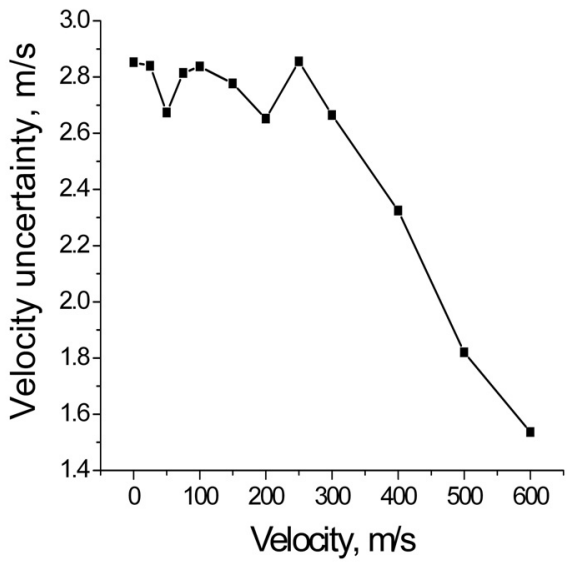

Fig. 20 Velocity uncertainty as a function of velocity magnitude based on measurements for the innermost fringe. The Rayleigh and reference signal amplitudes were maintained at $A_{f}=6000$ and $A_{R}=16000$, which were typical in these experiments.

\section{Conclusions and Future Work}

A Rayleigh scattering mass flux measurement technique has been developed using a solid etalon and direct imaging of a laser beam in the gas flow to provide multiple simultaneous measurement locations in the flow field. A Mach 0.56 free jet plume was studied to demonstrate feasibility of this technique. The low fringe orders where the fringe width was sufficiently sampled by the detector had good measurement accuracy, whereas the higher fringe orders 
with poor frequency resolution across the fringe width had poor measurement accuracy. The accuracy will be improved in the future by using a detector that can provide better spatial resolution across the fringe pattern. Ideally the detection system should provide a minimum of 3 pixels across the FWHM of the instrument function and at least 8 pixels across the FWHM of the Rayleigh fringe at any fringe location in the system to provide velocity, and hence mass flux measurements with acceptable accuracy. Several system improvements will be made in the future, including a new FP etalon that has a better quality reflectivity coating since unacceptable variations in the coating reflectivity were observed in the current etalon.

The next phase of this work involves installing the Rayleigh measurement system in the $15 \times 15 \mathrm{~cm}$ supersonic wind tunnel at NASA GRC to provide mass flux measurements. There are many anticipated technical challenges for that system integration, including the challenge of making measurements in the presence of shocks where the laser beam is refracted due to the sharp change in refractive index in the flow field, as well as the challenges associated with making optical measurements in an enclosed chamber. There will be image distortion issues related to imaging through the test section windows and stray light issues from the laser light scattered from the reflective tunnel walls. The next phase will be much more challenging than the current work where a low-speed flow in an open area was studied.

Plans for future work also include the development of a method to change the fringe locations rapidly to map out the measurement region with even higher spatial resolution. For a standard glass etalon the optical properties can be adjusted by changing the etalon temperature; hence changing the order of the interference pattern and the physical fringe locations. Temperature control of the etalon provides a slow time-response spatial scanning method for the etalon system. Liquid crystal Fabry-Perot (LCFP) etalons are now available in which the optical properties are changed by adjusting the voltage applied to the liquid crystal interface. A LCFP etalon with similar properties to the solid etalon is in production and will be characterized for use in this work when it arrives from the manufacturer. The LCFP etalon will allow Rayleigh measurements to be acquired at varying spatial locations across the image of the laser beam over a very short time period by rapidly scanning the voltage applied to the liquid crystal interface.

\section{Acknowledgments}

The authors would like to acknowledge Richard Seasholtz and Mark Wernet for their technical help in resolving issues encountered in this experiment. This work is supported by the Hypersonics discipline under NASA's Fundamental Aeronautics Program.

\section{References}

${ }^{1}$ Miles, R. B., Lempert, W. R., and Forkey, J. N., "Laser Rayleigh Scattering,” Meas. Sci. Technol., Vol. 12, No. 5, 2001, pp. R33-R51.

${ }^{2}$ Wang, G. H., Clemens, N. T., and Varghese, P. L., "Two-point, high-repetition-rate Rayleigh thermometry in flames: Techniques to correct for apparent dissipation induced by noise," Appl. Optics, Vol. 44, No. 31, 2005, pp. 6741-6751.

${ }^{3}$ Forkey, J. N., Lempert, W. R., and Miles, R. B., “Accuracy Limits for Planar Measurements of Flow Field Velocity, Temperature, and Pressure Using Filtered Rayleigh Scattering,” Exp. Fluids, Vol. 24, No. 2, 1998, pp. 151-162.

${ }^{4}$ Boguszko, M., and Elliott, G. S., "On the Use of Filtered Rayleigh Scattering for Measurements in Compressible Flows and Thermal Fields," Exp. Fluids, Vol. 38, No. 1, 2005, pp. 33-49.

${ }^{5}$ Seasholtz, R. G., Buggele, A. E., and Reeder, M. F., "Flow Measurements Based on Rayleigh Scattering and Fabry-Perot Interferometer," Optics and Lasers in Engineering, Vol. 27, No. 6, 1997, pp. 543-570.

${ }^{6}$ Vaughan, J. M., The Fabry Perot Interferometer, History, Theory, Practice, and Applications, Adam Hilger, Philadelphia, 1989, pp. 89-134.

${ }^{7}$ Mielke, A. F., Seasholtz, R. G., Elam, K.A., and Panda, J., “Time-average Measurement of Velocity, Density, Temperature, and Turbulence Velocity Fluctuations Using Rayleigh and Mie Scattering," Experiments in Fluids, Vol. 39, No. 2, 2005, 441454.

${ }^{8}$ Panda, J., and Seasholtz, R. G., "Velocity and Temperature Measurement in Supersonic Free Jets Using Spectrally Resolved Rayleigh Scattering," AIAA-99-0296, 1999.

${ }^{9}$ Seasholtz, R. G., Panda, J., and Elam, K. A., "Rayleigh Scattering Diagnostic for Measurement of Velocity and Density Fluctuation Spectra," AIAA-2002-0827, 2002.

${ }^{10}$ Mielke, A. F., Elam, K. A., and Sung, C. J., "Time-Resolved Rayleigh Scattering Measurements in Hot Gas Flows," AIAA2008-262, 2008. 
${ }^{11}$ Bivolaru, D., Danehy, P. M., Gaffney, R. L., and Cutler, A. D., "Direct-View Multi-Point Two-Component Interferometric Rayleigh Scattering Velocimeter," AIAA-2008-236, 2008.

${ }^{12}$ Lock, J. A., Seasholtz, R. G., and John, W. T., "Rayleigh-Brillouin Scattering to Determine One-Dimensional Temperature and Number Density Profiles of a Gas Flow Field," Applied Optics, Vol. 31, No. 15, 1992, pp. 2839-2848.

${ }^{13}$ Tenti, G., Boley, C., D., and Desai, R. C., "On the Kinetic Model Description of Rayleigh-Brillouin Scattering from Molecular Gases," Canadian Journal of Physics, Vol. 52, No. 4, 1974, pp. 285-290.

${ }^{14}$ Boley, C. D., Desai, R. C., and Tenti, G., "Kinetic models and Brillouin scattering in a molecular gas," Canadian Journal of Physics, Vol. 50, No. 18, 1972, pp. 2158-2173.

${ }^{15}$ Mielke, A. F., "Development of a Molecular Rayleigh Scattering Diagnostic for Simultaneous Time-Resolved Measurement of Temperature, Velocity, and Density," Ph.D. Dissertation, Department of Mechanical and Aerospace Engineering, Case Western Reserve Univ., Cleveland, OH, 2008.

${ }^{16}$ Whalen, A. D., Detection of Signals in Noise, Academic Press, New York, 1971, pp. 327-332. 